\title{
Lectura eficiente de un texto literario narrativo a partir de la metacognición en español como lengua no materna
}

\author{
Literatura-testu narratibo bat eraginkortasunez irakurtzea, ama-hizkuntza \\ ez den gaztelaniako metakogniziotik abiatuta \\ Efficient reading through metacognition: a narrative text in the context of \\ Spanish as a Second Language
}

\author{
María Luisa Aznar Juan \\ Universidad de Coímbra \\ aznarjuan1@gmail.com \\ https://orcid.org/0000-0003-2740-6329 \\ Elena Gamazo Carretero \\ Universidad de Coímbra \\ egc@uc.pt \\ https://orcid.org/0000-0002-5982-2055
}

Recibido / Noiz jaso den: 30/06/2021

Aceptado / Noiz onartu den: 23/10/2021

\begin{abstract}
Resumen
La compresión eficiente de textos no se restringe a la respuesta a preguntas sobre el contenido para comprobar qué han entendido los estudiantes. Para una comprensión lectora (CL) eficaz, es necesario activar estrategias de desenvolvimiento de la conciencia y control del aprendizaje. Los textos literarios narrativos accionan conocimientos lingüísticos, culturales y permiten representar situaciones comunicativas. Este trabajo se ocupa del desarrollo de la $\mathrm{CL}$ de un texto literario narrativo, desde una perspectiva instrumental, recurriendo a estrategias metacognitivas en el ámbito de la enseñanza de español como lengua extranjera (ELE). El objetivo es presentar los resultados de una investigación llevada a cabo con alumnos universitarios de nivel C1 y compararlos con los resultados de otra anterior efectuada en el nivel B2 dentro del mismo contexto. El método utilizado consiste en una investigación en el aula, aplicada y descriptiva, realizada a través del análisis cualitativo y cuantitativo simple de datos.
\end{abstract}

\section{Palabras clave}

Metacognición; estrategias; textos literarios narrativos; compresión lectora; español como lengua extranjera.

\section{Sumario}

1. INTRODUCCIÓN. 2. EL PROCESO LECTOR Y LAS ESTRATEGIAS DE COMPRENSIÓN DE TEXTOS LITERARIOS NARRATIVOS. 2.1. El proceso lector eficiente y los textos literarios narrativos. 2.2. La comprensión del texto literario narrativo desde la metacognición. 3. ESTUDIO DE CASO. 3.1. Hipótesis de partida y objetivos de la investigación. 3.2. Ámbito y metodología de la investigación. 3.3. Resultados y discusión. 4. CONCLUSIONES. REFERENCIAS. 


\begin{abstract}
Laburpena. Testuen ulermen eraginkorra ez da mugatzen edukiari buruzko galderei erantzutera ikasleek zer ulertu duten egiaztatzeko. Irakurmen eraginkorrerako, beharrezkoa da kontzientzia garatzeko eta ikaskuntza kontrolatzeko estrategiak aktibatzea. Literatura-testu narratiboek hizkuntza- eta kultura-ezagutzak sortzen dituzte, eta komunikazio-egoerak irudikatzeko aukera ematen dute. Lan hau literatura-testu narratibo baten irakurmena garatzeaz arduratzen da, ikuspuntu instrumental batetik, eta espainiera atzerriko hizkuntza gisa duen esparruko estrategia metakognitiboetara jotzen du. Helburua da C1 mailako ikasle unibertsitarioekin egindako ikerketa baten emaitzak aurkeztea eta B2 mailan testuinguru berean egindako beste ikerketa baten emaitzekin alderatzea. Metodoa ikasgelako ikerketa aplikatu eta deskriptibo bat $\mathrm{da}$, datuen analisi eta kualitatibo eta kuantitatibo bakunaren bidez.
\end{abstract}

Gako hitzak. Metakognizioa; estrategiak; litteratura-testu narratiboak; irakurmena; espainiera atzerriko hizkuntza gisa.

\begin{abstract}
Reading comprehension assessment for students is not limited to the question and answer strategies. In order to achieve an effective reading comprehension ( $\mathrm{RC}$ ) it is paramount to activate the conscious awareness and learning control strategies. In this context, literary texts are not only a source of linguistic and cultural knowledge, but they are also an optimal framework for communicative situation representation. This study focuses on the RC development in literary texts using metacognitive strategies for the Spanish as a foreign language (ELE) classroom. Our aim is to present the findings of a research carried out with C1 level university students and showcase its connection with the results of a previously conducted research with B2 level students in the same context. The used methodology entails a classroom based research, both applied and descriptive, completed through a simple qualitative and quantitative data analysis.
\end{abstract}

Keywords. Metacognition; reading comprehension; strategies; literary text; foreign languages.

\section{Introducción}

La mayor parte de las actividades académicas y sociales se realiza a través de la lectura. Leer es un acto que nos permite acceder a un universo de información necesario tanto para aprender, como para relacionarnos con el medio. De ahí que el desarrollo de la competencia lectora tenga un efecto directo en la formación integral de los discentes. Por este motivo «independientemente de sus aspiraciones académicas o laborales, la competencia lectora de los alumnos es importante para su participación activa en la comunidad y para su vida económica y personal» (OCDE, 2017, p. 36).

Ahora bien, los docentes de lenguas extranjeras nos encontramos con numerosas situaciones en las que los estudiantes entienden el significado global de un texto. Sin embargo, cuando se les plantean preguntas específicas sobre lo que han leído, no responden correctamente. Esto demuestra que, aún hoy, la percepción de la lectura y de la comprensión lectora por parte de los alumnos se limita, en la mayoría de las ocasiones, a un ejercicio de descodificación o de entendimiento superficial del texto. En estos casos nos hallamos ante lectores poco eficientes que no son capaces de activar habilidades estratégicas para obtener una percepción completa del texto más allá de su significado explícito. 
Los estudios sobre metacognición lectora revelan que la enseñanza explícita de las estrategias metacognitivas mejora significativamente el nivel de compresión del discurso escrito (Brown, Palincsar \& Ambruser, 2006). Además, permite desarrollar habilidades del pensamiento relacionadas con la reflexión, el control y el autoaprendizaje. En general, la instrucción metacognitiva favorece el desenvolvimiento de la capacidad intelectual y del pensamiento crítico, estratégico y creativo para formar lectores competentes. En este sentido, consideramos que leer no solo consiste en reconocer y descodificar la información. Leer también supone pensar, reflexionar, sentir, imaginar, conectar conocimientos y relacionar nuestra realidad con la representada en el texto.

Según el Marco común europeo de referencia para las lenguas, el recurso a los textos literarios en el aula adquiere relevancia no solo por su función estética, sino también porque «cumplen muchos más fines educativos, intelectuales, morales, emocionales, lingüuísticos y culturales que los puramente estéticos» (2002, p. 60). Además, las obras literarias, en especial las narrativas, promueven la creación de inferencias deductivas y la activación de los conocimientos previos del lector para construir nuevos significados y alcanzar el entendimiento eficiente del discurso escrito.

Consecuentemente, se defiende el uso de los textos literarios narrativos de mayor envergadura y extensión en niveles intermedios y avanzados de ELE (español como lengua extranjera), pues en estos casos los estudiantes ya disponen de las herramientas lingüuísticas necesarias para desarrollar la lectura desde una perspectiva metacognitiva.

Por consiguiente, en este trabajo se exponen los resultados de una investigación cualitativa y cuantitativa simple sobre el uso de las estrategias metacognitivas en la compresión lectora de un texto literario narrativo, desde una perspectiva instrumental de la lengua, de estudiantes con un nivel C1 de español como lengua extranjera. El propósito principal es presentar los datos contrastándolos con la información obtenida de un trabajo desarrollado en circunstancias similares con estudiantes de nivel B2 de ELE.

Así pues, en las páǵinas que siǵuen se abordan alǵunas cuestiones teóricas relacionadas con el proceso lector y las estrategias de comprensión de textos literarios. Para tal, se refieren por separado los procedimientos que intervienen en la lectura eficiente de textos literarios y la comprensión del texto literario desde la metacognición. A continuación, se presenta el estudio de caso, donde se especifica, por un lado, la metodología de la investigación y, por otro, los resultados y la discusión de estos. Por último, se exponen las conclusiones y las referencias bibliográficas. Esperamos que este trabajo sirva para crear actividades que permitan mejorar la compresión lectora a través de la enseñanza explícita de las estrategias metacognitivas para, de este modo, crear lectores estratégicos y competentes en ELE. 


\section{El proceso lector y las estrategias de comprensión de textos literarios narrativos}

\subsection{El proceso lector eficiente y los textos literarios narrativos}

Actualmente existe un consenso general en aceptar el acto de leer como un proceso cognnitivo complejo (Puente, 1991; Binkley, Rust \& Williams, 1997; Zwaan \& Singer, 2003), frente a la idea basada principalmente en la descodificación de palabras. Autores como Perfetti (2007) o Cerchiaro et al. (2011, p. 102) sitúan el cambio de perspectiva sobre la lectura en los años 70 y 80 del siǵlo XX. La evolución de las corrientes cognitivistas, junto con el desarrollo de los estudios sobre la CL en el ámbito de la linguuística, cuyo objetivo principal era conocer las estrategias y procesos desarrollados por el lector competente, fueron los pilares clave de esta transformación.

La concepción de la lectura basada en este nuevo punto de vista realza la figura del receptor como agente activo, capaz de desenvolver habilidades y estrategias que le permiten movilizar sus propias experiencias para alcanzar la comprensión integral del texto. En este sentido las piezas fundamentales en el entendimiento del discurso escrito son el lector, el escritor y el texto (Puente, 1991; Solé, 1994). La reciprocidad creada entre los conocimientos y la información transmitida por el autor permite la construcción de nuevos significados que posibilitan el reconocimiento del texto, más allá de la descodificación o la literalidad de palabras y frases.

Durante la creación de significados el sujeto relaciona sus ideas sobre el mundo con los datos de lo que se lee. Para evitar esta situación, es necesario controlar la CL utilizando estrateǵias de regulación, control y evaluación. En este sentido, Aragón de Moreno (2014, p. 42) defiende que leer es una acción estratégica, constructiva, interactiva y metacognitiva.

Desde la perspectiva que acabamos de exponer, mantenemos que el transcurso de la lectura es un proceso activo y multidimensional en el que convergen los procedimientos cognitivos del pensamiento y los elementos lingüústicos. Consecuentemente, el buen lector «debe identificar palabras, detectar estructuras sintácticas, conectar entre sí las diversas partes del texto y relacionarlas con su propio conocimiento general» (Irrazábal \& Sáux, 2005, p. 35). Así pues, como mantiene el Marco de Evaluación y de Análisis de PISA para el Desarrollo: Lectura, matemáticas y ciencias (OCDE, 2017, p 34), los componentes esenciales de la lectura son el significado de las palabras, el procesamiento de frases y la comprensión eficaz, además de los elementos del alfabeto y la comprensión oral básica.

Ahora bien, el objetivo más inmediato del acto de leer es la comprensión lectora, entendida como una actividad interactiva de construcción de conoci- 
mientos que se practica con objetivos específicos. La consecución de los objetivos se logra por el recurso a estrategias que permiten monitorizar y evaluar las acciones desarrolladas a lo largo de la lectura. A su vez, estas acciones se suceden de manera progresiva y su éxito depende no solo de la información o las operaciones formales de la lengua, sino también de la interacción entre los esquemas del receptor, el texto y los contenidos lingüísticos y extralingüuísticos (Cerchiaro et al., 2011). En este sentido podemos afirmar que el entendimiento «surge de la mente del lector, quien usa su conocimiento para interpretar e integrar lo que lee» (Irrazábal \& Sáux, 2005, p. 34).

Las fases de la CL, según Puente et al. (2019, p. 23-24), se dividen en cuatro bloques. El primero está relacionado con el contenido de las estructuras del conocimiento que permiten realizar inferencias deductivas. El segundo tiene que ver con el vínculo que se establece entre el texto y el receptor cuando este último infiere el significado de lo que el primero procura comunicar. El tercer apartado se refiere al uso de las estrateǵias que permiten deducir significados y, por último, el cuarto bloque corresponde a la representación mental del discurso.

En suma, de lo expuesto hasta el momento, concluimos que la formación del lector competente depende del desarrollo efectivo de la CL, entendida como un procedimiento en el que los diferentes componentes y agentes que intervienen en la lectura actúan en conjunto. Por consiguiente, para estimular la comprensión discursiva escrita eficiente es necesario poseer la capacidad de relacionar los conocimientos previos con los datos del texto para crear nuevos significados (Calero, 2012; Richter \& Rapp, 2014); plantear las metas de la lectura; escoger estrateǵias cognitivas y metacognitivas que permitan una interpretación correcta; reflexionar sobre el desarrollo y la evolución del proceso lector; percibir posibles fallos y elegir las estrategias adecuadas para su reparación (Romero et al., 2018; Puente et al., 2019). Además, es transcendental saber diferenciar los datos relevantes de los menos importantes; sintetizar la información (Vidal-Abarca et al., 2010; Puente et al., 2019); hacer deducciones y formular preguntas (Puente et al., 2019). En definitiva, como señala González Fernández (1992, p. 164), la práctica eficaz de la lectura depende de sujetos que revelan una adecuada capacidad de supervisión; un mayor dominio en el empleo de estrategias de aprendizaje y facilidad para recurrir a estrategias alternativas cuando es necesario reparar la actividad lectora.

Por el contrario, son rasgos característicos de un lector poco competente la incapacidad para relacionar y contrastar sus pensamientos y sensaciones con la información proporcionada en el texto (Calero, 2012); la falta de objetivos; la escasez de estrateǵias cognitivas y metacognitivas; la poca o ninguna reflexión sobre el desarrollo y la evolución de su proceso y la dificultad para reconocer fallos y repararlos. Además, estos lectores muestran limitaciones a la hora de diferenciar los datos relevantes de los menos importantes; les cuesta sintetizar 
la información y deducir. En definitiva, se trata de sujetos que «usualmente no participan activa ni estratégicamente, no disponen de estrateǵias adecuadas, o no las usan, o, al usarlas, no lo hacen flexiblemente en un intento de ajustarlas a la consecución de sus metas. Se limitan, entonces, a la mera decodificación del texto» (López Jiménez \& Arciniegas Lagos, 2004, p. 26).

La definición y caracterización de lectura y CL expuesta en las líneas que preceden, así como las cualidades aludidas sobre la práctica de lectura eficaz se adaptan, de modo generalizado, a todos los idiomas. De ahí que apliquemos los presupuestos descritos en este apartado al español como segunda lengua o lengua extranjera.

Por lo que se refiere al ámbito de ELE, la comprensión lectora ocupa un lugar relevante en el Marco común europeo de referencia para las lenguas (2002) que, a su vez, recomienda de forma evidente la instrucción basada en estudios de carácter literario. Entre los beneficios de la CL de textos literarios en el aula de español, destacamos, por un lado, el hecho de permitir el desarrollo de competencias lingüuísticas, culturales, sociales y éticas y, por otro, la capacidad de crear el hábito de leer. Al mismo tiempo, la instrucción literaria de CL se convierte en una práctica motivadora para los estudiantes por la relación que se establece entre el lector, la historia y los personajes (Albaladejo García, 2007, p. 8) y por tratarse de un material auténtico. Así pues, consideramos que las posibilidades de interpretación y relación con la realidad que la literatura ofrece favorecen la reflexión, el debate y la construcción de conocimientos (Aznar Juan \& Gamazo Carretero, 2019, p. 18) y, en nuestro trabajo, defendemos el recurso a los textos literarios, específicamente, los de carácter narrativo, como medio de instrucción y entendimiento del discurso escrito.

Por último, en lo que concierne a la narración, somos de la opinión de que la propia acción narrativa estimula la creación de un número más elevado de inferencias predictivas, ya que el lector es capaz de imaginar lo que va a suceder en la historia. Por consiguiente, centramos nuestra investigación en la comprensión de textos narrativos desde la perspectiva de la lingüústica cognitivista ${ }^{1}$, con el objetivo de crear lectores eficientes capaces de desarrollar habilidades metacognitivas que les permitan reflexionar, supervisar y controlar su actividad lectora.

1 El estudio que presentamos se ocupa del desarrollo de la CL a partir de un texto narrativo como instrumento para el aprendizaje de ELE en un contexto de no inmersión. Por este motivo, nos basamos en los estudios provenientes de la Lingüuística Cognitiva y no de la Literatura Cognitiva. No obstante, podemos señalar alǵunos autores de referencia sobre Literatura Coǵnitiva como Nikolajeva, M. (2014); Pérez Jiménez, E. (Ed.) (2015); Zunshine, L. (Ed.) (2015) y Colomer, T., Manresa, M., Ramada Prieto, L. y Reyes López, L. (2018). 


\subsection{La compresión del texto literario narrativo desde la metacognnición}

Atendiendo a lo expuesto en el apartado anterior, la comprensión eficaz del texto literario depende de la capacidad de relacionar conocimientos, reflexionar sobre el decurso de la lectura y utilizar estrategias que permitan controlar y regular la práctica lectora. En este contexto, adquiere relevancia la metacognición, entendida como «el conocimiento que uno tiene sobre los procesos y productos cognitivos o sobre cualquier cosa relacionada con ellos, es decir, las propiedades de la información o los datos relevantes para el aprendizaje» (Flavell, 1997, p. 232).

Según esta definición, la función metacognitiva es fundamental para que el alumno-lector cumpla las metas propuestas de forma consciente y autónoma. De esta manera, el profesor, en su labor de mediador, consigue que los discentes asuman un papel activo en la construcción de sus propios saberes. Además, logra formar lectores inteligentes capaces de desenvolver el gusto por leer literatura, al mismo tiempo que desarrollan las competencias lingüústicas, sociales, éticas, culturales y académicas.

La metacognición, aplicada a las segundas lenǵuas o lenǵuas extranjeras, promueve el uso de estrategias que ayudan a los alumnos a conseguir sus metas durante el aprendizaje del idioma y logra que la comprensión discursiva sea provechosa. Por ello, somos de la opinión de que los docentes deben promover la CL de textos literarios desde la metacognición, para crear lectores capaces de aproximarse a la lengua de un modo reflexivo y autónomo. Por consiguiente, en nuestro trabajo partimos del presupuesto de que la metacognición y el dominio de las estrategias metacognitivas tienen un peso fundamental en el aprendizaje de la L2 y la LE (Nunan, 1996; Sandoval Zúñiga et al., 2010). Como consecuencia, defendemos la necesidad de proporcionar al estudiante una instrucción explícita en los procesos y estrategias metacognitivas de CL.

A ǵrandes rasǵos, definimos las estrateǵias metacoǵnitivas como acciones que el receptor del discurso escrito utiliza de manera intencional y consciente para la autorregulación, autocontrol y autoevaluación de la práctica de la lectura (Richards, Platt \& Platt 1997; Solé, 1998; Ramírez Peña et al., 2015).

Existe el consenso entre gran parte de los estudios de metacognición en aceptar tres fases fundamentales en los procesos metacognitivos lectores, a las que se les asocian estrategias concretas (Baker \& Brown, 1984; Richards \& Lockart, 1998 o Heit, 2012): planificación (antes de la lectura), supervisión (durante la lectura) y evaluación (después de la lectura). Las actividades de planificación tienen como objetivo preparar la interpretación textual a través de su contextualización y, al mismo tiempo, incentivar la curiosidad y el interés (López González, 2020). Para estos efectos, es necesario establecer los propósitos y definir las estrategias que permitirán alcanzar las metas propuestas. Gutiérrez-Braojos \& Sal- 
merón Pérez (2012, p. 185) presentan como estrategias principales de esta fase la identificación del género discursivo, señalando su estructura y características; el establecimiento de la finalidad lectora, esto es, si por ejemplo se lee para tener una visión global del texto, extraer ideas principales o responder a preguntas; la activación de conocimientos previos para reconocer e integrar la información nueva, deducir y realizar predicciones y, por último, generar preguntas relacionadas con el fraǵmento u obra que trabajará.

La supervisión se realiza durante la lectura y consiste en ejecutar tareas o actividades que permiten crear una imagen mental de lo que se ha leído, tener conciencia de si la comprensión se está efectuando de forma correcta o no y, en caso de que existan errores de entendimiento, utilizar los medios de reparación convenientes. Las estrateǵias metacognitivas más usadas durante esta etapa consisten en tomar notas; hacer preguntas; subrayar; parafrasear; resumir; releer; identificar los elementos clave del texto; anticipar información; deducir, predecir y hacer hipótesis; organizar y localizar la información; ordenar el texto; identificar las dificultades y reparar los fallos (López Jiménez \& Arciniegas Lagos, 2004; Aznar Juan \& Gamazo Carretero, 2019).

La evaluación supone reflexionar sobre el procedimiento de comprensión del discurso y su eficacia. Durante este periodo, el estudiante hace una valoración retrospectiva en la que relaciona las metas propuestas en la fase de planificación y los logros obtenidos. En esta fase es importante reflexionar y evaluar el nivel alcanzado y la fiabilidad de las estrategias utilizadas. Para ello, los discentes pueden discutir y formularse preguntas sobre su propio aprendizaje.

Por lo que se refiere al proceso de instrucción del alumno para incentivar el desarrollo de las habilidades y estrategias metacognitivas, según López Delǵado (2005, p. 86), el profesor tiene que explicar, en primer lugar, qué es lo que se va a aprender; en seǵundo lugar, ha de modelar la estrateǵia y, por último, iniciará la práctica guiada. En otras palabras, para que el resultado de la instrucción sea provechoso es imprescindible explicar en qué consiste la estrategia; qué utilidad y beneficios aporta; cómo, cuándo y dónde se puede utilizar y cómo evaluar el resultado de su uso (Winogard \& Hare, 1988; López Delǵado, 2005).

Las principales estrateǵias metacognnitivas consideradas en nuestro trabajo son similares a las referidas en el estudio sobre metacognición que propusimos para el nivel intermedio de ELE, lo que nos permite establecer la comparación entre los resultados de esta investigación en relación con la anterior. Así pues, basándonos en los planteamientos de Delmastro (2010), Moreno (2011) y Gutiérrez-Braojos \& Salmerón Pérez (2012), a continuación exponemos las estrategias seleccionadas para la CL de textos literarios presentadas por Aznar Juan \& Gamazo Carretero (2019, p. 19-20). 
Entre las estrategias metacognitivas antes de la lectura o de planificación, destacamos las siguientes:

- Utilizar planificadores y activadores previos de la actividad de aprendizaje.

- Determinar las metas, objetivos y la finalidad de la lectura.

- Determinar las acciones necesarias para el cumplimiento de la tarea.

- Estructurar el trabajo y definir las estrategias de lectura.

- Establecer las condiciones adecuadas para el aprendizaje.

- Activar conocimientos previos.

- Relacionar con lo conocido.

- Preparar los componentes lingüuísticos y paralingüústicos para la tarea.

- Determinar y reconocer las estructuras textuales.

- Hacer hipótesis y/o predicciones sobre el contenido.

- Generar preguntas.

Entre las estrategias metacognitivas durante la lectura o de supervisión, destacamos las siguientes:

- Contestar a preguntas sobre el texto.

- Generar nuevas preguntas.

- Detectar información relevante y secundaria.

- Identificar y analizar errores de interlengua en la propia producción y la de los compañeros.

- Identificar palabras o estructuras que necesitan ser aclaradas.

- Parafrasear, resumir y sintetizar.

- Realizar nuevas inferencias deductivas y predicciones.

Entre las estrategias metacognitivas tras la lectura o de control, destacamos las siguientes:

- Verificar la producción según parámetros propios.

- Responder a preguntas sobre el texto, evaluando lo aprendido.

- Resumir y esquematizar la información del texto para evaluar lo aprendido.

- Responder a preguntas sobre las estrategias utilizadas.

- Formular preguntas entre los estudiantes.

- Cuestionar estrategias en la toma de decisiones y la solución de problemas.

- Cuestionar nuestra relación con el proceso de lectura.

- Cuestionar nuestro aprendizaje/autoaprendizaje.

En suma, por lo expuesto, concluimos que el recurso a las estrategias metacognitivas es fundamental para la apreciación real del discurso, entendida como un acto multidimensional, constructivo y activo. Por esta razón, consideramos que el docente desempeña un papel relevante como orientador del estudiante a lo largo de la CL. De ahí la necesidad de establecer pautas de instrucción explícitas que guíen a los alumnos. Así pues, en la investigación planteada en este trabajo 
tenemos en cuenta las fases del proceso lector descritas y las estrategias señaladas para cada una de esas etapas. Además, consideramos las indicaciones sobre el procedimiento de enseñanza/aprendizaje de las estrategias metacognitivas.

\section{Estudio de caso}

\subsection{Hipótesis de partida y objetivos de la investigación}

La preocupación sobre el estado y el desarrollo de la compresión lectora en los estudiantes de niveles de enseñanza intermedio y superior se ha incrementado de manera notable a lo largoo de esta última década. La concepción de la lectura y su comprensión ha pasado de ser considerada un acto casi mecánico de descifrado a un acto de razonamiento y reflexión. La lectura entendida desde esta última perspectiva se convierte en un proceso de comprehensión e interacción entre el emisor y el receptor del texto en el que se establece el vínculo entre los conocimientos y la realidad del lector y los conocimientos transmitidos por el autor a través de la escritura. El resultado de esta unión supone para el lector adquirir nuevos conocimientos y, al mismo tiempo, desarrollar valores éticos, culturales y sociales.

Así, entender el texto de la manera que acabamos de exponer implica extraer el sentido ǵlobal del mensaje por medio del análisis, la observación y la reflexión. De este modo, al ejercitar al alumno para una CL eficaz, también le estamos preparando para que pueda aplicar las estrategias adquiridas a diferentes ámbitos de su vida cotidiana o profesional. Por tanto, podemos afirmar que al formar lectores competentes estamos formando ciudadanos cualificados, aptos para desenvolverse en las distintas esferas de la sociedad.

Programas como PISA (Programme for International Student Assessment) nos ofrecen, desde el 2000, informes cada tres años sobre el estado y evolución de la lectura en la enseñanza obligatoria. Sin embargo, no disponemos de datos recogidos sistemáticamente sobre la lectura en el ámbito del español como segunda lengua o lengua extranjera. Por ello, consideramos necesario desenvolver investigaciones que ofrezcan resultados más precisos sobre la práctica de la compresión lectora. En este sentido, el objetivo principal de nuestro trabajo es hacer un diaǵnóstico, sobre la CL de un texto narrativo literario desde una perspectiva metacognitiva e instrumental, que permita proponer actividades adecuadas para el desarrollo de la comprensión lectora en ELE dentro del ámbito universitario.

Para dar cumplimiento a este objetivo, partimos de la hipótesis de que la compresión lectora es considerada por los estudiantes de lengua materna portuguesa una actividad fácil y, por tanto, queda relegada a un segundo plano a lo largo del transcurso escolar. Asimismo, observamos que los alumnos de nivel C1 de ELE presentan dificultades a la hora de responder a preguntas que implican 
una reflexión sobre el contenido principal o secundario de los textos y su estructura. De ahí que pensemos que pueda existir algún fallo en el entrenamiento de los discentes en relación a las estrategias metacognitivas de compresión lectora.

Por tanto, a partir de esta hipótesis, nos planteamos las siguientes cuestiones: ¿Qué tipo de formación recibe el alumno de nivel C1 en relación al proceso de compresión lectora en ELE? ¿La práctica de la CL es habitual en el aula? ¿Con qué frecuencia se trabaja este tipo de destreza en las clases? ¿El alumno de nivel C1 posee consciencia de los procesos y estrategias metacognitivas aplicadas a la CL? ¿Qué estrategias metacognitivas son las más utilizadas para la adquisición de la comprensión lectora? ¿Existe autorregulación en el proceso de CL? ¿Cómo se materializa la autorregulación? ¿Qué tipo de textos son los más utilizados? ¿Cómo se explotan los textos narrativos literarios? ¿El desarrollo de las estrategias metacognitivas depende del nivel y la madurez lingúística y coǵnitiva de los estudiantes?

La formulación de estas preguntas responde a los siguientes objetivos específicos de la investigación:

- Verificar con qué frecuencia se trabaja la CL en el aula.

- Verificar el nivel de consciencia de los estudiantes con respecto a los procesos y estrategias metacognitivas aplicadas a la CL.

- Observar hasta qué punto el alumno es capaz de planificar, controlar y evaluar los resultados del aprendizaje adquiridos en la compresión lectora.

- Comprobar el desarrollo de las estrateǵias metacognitivas según el nivel y la madurez lingüuística y cognitiva de los alumnos.

En síntesis, el estudio de caso que presentamos en este trabajo, además de hacer el diaǵnóstico sobre el conocimiento de las estrategias metacognitivas de CL en el nivel C1 de ELE, establece una comparación entre los resultados obtenidos en esta investigación y los procedentes de un trabajo anterior realizado en el mismo contexto educativo con alumnos de nivel B2.

\section{2. Ámbito y metodología de la investigación}

El trabajo que presentamos consiste en una investigación en el aula, descriptiva y aplicada, en la que realizamos un análisis cualitativo y cuantitativo simple de datos sobre el uso de las estrategias metacognitivas en la CL de un texto literario narrativo en un nivel C1 de español como lengua extranjera ${ }^{2}$. El ámbito del

2 Recordamos que centramos nuestro estudio en la lectura de un texto literario narrativo con una finalidad instrumental y de aprendizaje de ELE. Por este motivo, no nos ocupamos de aspectos relacionados con los procesos complejos de interpretación, ni de la vivencia y extrañamiento estético de los textos literarios. 
estudio se enmarca en el área de la didáctica específica, concretamente, en el contexto de las metodologías aplicadas a la adquisición y aprendizaje de segundas lenguas.

El corpus de los datos fue recogido durante el sexto semestre de lengua española. Para ello, contamos con un universo de 72 informantes (44 mujeres y 28 hombres) y 144 cuestionarios. Los informantes procedían de dos grupos distintos con el mismo nivel de enseñanza: un grupo de 35 estudiantes y otro de 37 . Los criterios para la selección de la población objeto de estudio fueron, por un lado, que se tratase de alumnos de nacionalidad portuguesa, excluyendo a los que se encontraban en el programa de intercambio Erasmus; por otro, que estuviesen matriculados en la variante español/portuǵués o español/inglés del grado en Lenguas Modernas de la Facultad de Letras de la Universidad de Coímbra (FLUG) y que tuviesen un nivel C1 de español.

Por lo que se refiere a la metodología, Moreno Fernández (1990, p. 39) mantiene que toda investigación tiene que considerar qué se va a hacer, para qué y cómo hacerlo. Estas condiciones suponen el establecimiento de la hipótesis de trabajo, los objetivos, la entidad social, la recogida, preparación y análisis de los datos y, por último, la presentación de los resultados. Así pues, en el estudio que proponemos seguimos las indicaciones de Moreno Fernández (1990) y, tras el establecimiento de los objetivos, la hipótesis de partida y la entidad social, elaboramos dos cuestionarios cerrados cuyas respuestas fueron analizadas posteriormente.

Los cuestionarios constan de dos partes: en la primera, se recogen los datos de los sujetos encuestados y se expone brevemente el motivo de la investigación. La segunda parte comprende doce preguntas relacionadas con la compresión lectora, las estrategias metacognitivas y el recurso a los textos literarios en el aula de ELE. El tipo de respuestas son de selección múltiple o valoración según criterios de ordenación del 1 al 5, siendo 1 la puntuación mínima y 5 la máxima.

Para la aplicación de los cuestionarios, dividimos el proceso de la investigación en cinco fases. Durante la primera, se recopiló la información extraída del Cuestionario 1. A continuación, se examinaron los resultados; seguidamente, se procedió a la exposición docente; después, se recogieron los datos del Cuestionario 2 ; y, por último, se analizaron y compararon los elementos obtenidos.

1. ${ }^{a}$ Fase. Cuestionario 1.

2. ${ }^{a}$ Fase. Análisis.

3. ${ }^{\mathrm{a}}$ Fase. Exposición docente.

4. ${ }^{a}$ Fase. Cuestionario 2.

5. ${ }^{a}$ Fase. Análisis y comparación de los resultados.

El objetivo de la primera y cuarta etapa fue recoger datos para comprobar la relación de los discentes con la compresión lectora de textos literarios. El propósito 
de la tercera fase fue desarrollar una propuesta didáctica en la que se trabajasen explícitamente las estrategias metacognitivas de CL durante las etapas de planificación, supervisión y evaluación. El trabajo fue desarrollado a lo largó de cuatro clases presenciales de 120 minutos y tres sesiones asincrónicas en la plataforma digital de la FLUC. La primera y segunda sesión estuvieron dedicadas a la planificación; la tercera, cuarta y quinta, a la supervisión; y la sexta, a la evaluación.

La elección de las estrategias metacognitivas que inteǵran el estudio dependió de la madurez y el perfil de los grupos de informantes de la muestra. Este criterio fue el mismo que seguimos para seleccionar la obra literaria en la que basamos nuestra unidad didáctica: El tiempo entre costuras, de María Dueñas. La elección de esta novela se debe a dos factores: en primer lugar, los alumnos conocían el contexto político y social en el que está ambientada la obra, motivo por el cual consideramos que tendrían mayor facilidad en comprenderla y aumentar su interés por la lectura. Por otra parte, el nivel lingüústico de este texto narrativo era adecuado a los conocimientos de lenǵua española que poseían los estudiantes.

En la primera sesión presencial de la etapa de planificación, definimos, junto con los alumnos, los objetivos de aprendizaje y la finalidad de la lectura; determinamos las acciones necesarias para realizar la tarea y su estructura; explicamos en qué consistían las estrategias metacognitivas que iríamos a aplicar; qué utilidad y beneficios aportaban; cómo, cuándo y dónde se podían utilizar y cómo evaluar el resultado de su uso (Winogard \& Hare, 1988; López Delǵado, 2005). Una vez que los estudiantes tomaron conciencia de la manera en que se desarrollaría la CL y qué estrategias emplearían, pasamos a activar sus conocimientos previos, relacionándolos con los de su propia lenǵua y cultura. A continuación, realizamos una actividad de predicción del contenido a partir del título del libro y los elementos paralingüuísticos de la portada, que supuso una primera toma de contacto con la obra y las expectativas sobre la lectura.

La segunda sesión de prelectura fue asincrónica y sirvió para preparar los componentes lingüuísticos relacionados con la CL; aclarar conceptos y aspectos textuales que ayudarían a la lectura; reconocer la estructura narrativa y generar preguntas hipotéticas sobre la trama, tras haber escuchado las opiniones del resto del grupo sobre el arǵumento del libro, basadas en la información de la portada.

La etapa de supervisión o control comenzó en la tercera semana y se extendió hasta la sexta. Cada sesión de esta fase correspondió a la lectura de una parte del libro, según la estructura de la obra. Decidimos que la primera sesión y la última fuesen presenciales coincidiendo con el inicio y el fin de la narración. Así, cuando empezamos la primera sesión, los alumnos ya habían leído hasta la primera parte, lo que nos permitió realizar tres actividades distintas, de manera individual, por parejas y en grupos. A lo largo de toda la propuesta didáctica, utilizamos la plataforma Padlet para registrar y, posteriormente, analizar las respuestas de los estudiantes. 
La primera actividad de la sesión 3 consistió en plantear preguntas de comprensión entre los compañeros. El siguiente ejercicio se realizó en parejas y supuso detectar la información relevante y secundaria del texto. Por último, en grupos de cuatro o cinco estudiantes se identificaron las palabras o estructuras que necesitaban ser aclaradas o que suponían alguna dificultad para la compresión discursiva.

Las sesiones 4 y 5 transcurrieron de forma asincrónica e individual, a través de la plataforma digital de la FLUC. Los alumnos tuvieron que realizar cuatro tareas en las que parafrasearon y sintetizaron las partes del texto señaladas por el docente; reescribieron algunos fraǵmentos de los capítulos leídos reproduciendo las ideas principales; relacionaron datos de la lectura con palabras concretas y anticiparon información, realizando nuevas deducciones y predicciones sobre la última parte del libro.

La última sesión de la supervisión fue presencial; en ella se hizo un repaso y análisis del trabajo desarrollado en las clases asincrónicas, se generaron nuevas preguntas sobre la última parte del libro que se había leído y se comprobaron las hipótesis enunciadas durante la fase de preparación.

La supervisión de la CL estuvo dedicada a la etapa de evaluación, donde los estudiantes a través de preguntas formuladas por las docentes y por ellos mismos, discutieron y evaluaron lo aprendido y reflexionaron sobre la eficacia de las estrategias empleadas. Terminamos el proyecto de compresión lectora con la opinión personal de los alumnos sobre el desarrollo del proceso lector y la lectura del texto narrativo El tiempo entre costuras.

Tras la tercera fase de la investigación, los discentes respondieron de forma individual y anónima al cuestionario 2, cuyos resultados fueron analizados y comparados con los obtenidos en una investigación similar aplicada a un nivel intermedio de ELE. Así pues, a continuación, presentamos los resultados y la discusión de los datos obtenidos a partir de la aplicación de las diferentes etapas de la investigación.

\subsection{Resultados y discusión}

La primera fase de la investigación consistió en la contextualización y aplicación del Cuestionario 1. Explicamos a los estudiantes que se trataba de una tarea anónima y no obligatoria cuyas respuestas tenían que considerar el aprendizaje de años anteriores.

A la primera pregunta del cuestionario, 32 informantes respondieron que consideraban la expresión oral la destreza más practicada; 28 seleccionaron la expresión escrita; 10, la comprensión lectora y 2, la auditiva. Un total de 68 alumnos calificaron como monótono y aburrido el proceso de comprensión lectora. Los 4 restantes no respondieron. 
Respecto al tipo de textos que se emplean con más frecuencia para practicar la CL, 22 informantes declararon que eran los divulgativos; 15 los argumentativos; 13 los narrativos y 11 los expositivos. Los menos habituales eran los científicos (2) y los publicitarios y literarios (3 y 6).

En cuanto a los textos literarios, los extraídos de blog y plataformas digitales son los más populares (33). Dentro de los narrativos, son más comunes los relatos breves o cuentos (22) que los extensos o novelas (7). El género poético no fue referenciado.

La mayoría de los estudiantes confirmaron que no habían utilizado estrategias de CL (62). Sin embargo, 6 informantes manifestaron haber usado alguna estrategia y 4 no contestaron.

Las respuestas a la pregunta número seis reflejaron que las actividades de prelectura no se suelen plantear en las clases, puesto que 58 informantes declararon que nunca las habían realizado y 12 en algunas ocasiones. Entre ellas, destacaron, por orden de frecuencia: hacer hipótesis y/o predicciones sobre el contenido (5); relacionar los textos con lo conocido (3); determinar y reconocer las estructuras textuales (2); y activar conocimientos previos (2).

Durante la lectura, casi la totalidad de los informantes (67) revelaron que, en algunas ocasiones, sí habían hecho actividades de control. Las más habituales eran: identificar palabras o estructuras que necesitasen ser aclaradas (31); contestar a preguntas sobre el texto (16); parafrasear, resumir y sintetizar (11); y detectar información relevante y secundaria (9).

Las actividades de poslectura son las más frecuentes (70); entre ellas, las más habituales fueron: verificar la producción según parámetros propios (23); responder a preguntas sobre el texto, evaluando lo aprendido (21); resumir (18) y esquematizar la información para evaluar lo aprendido (18).

Por último, los informantes respondieron que los contenidos más trabajados en la CL son el léxico y el cultural, aunque un número importante de discentes destaca que la lectura les ayuda a reflexionar y a ser más creativos.

Tras la exposición didáctica docente (Fase 3), los estudiantes respondieron al Cuestionario 2. Sobre las preguntas relacionadas con la percepción de la CL, 53 alumnos dijeron que les parecía un proceso dinámico, mientras que 10 siǵuen considerándolo aburrido.

Entre las actividades de prelectura más útiles, se destacan, en primer lugar, estructurar el trabajo, definiendo las estrategias de lectura (43); en segundo lugar, preparar los componentes linguiísticos y aclarar aspectos textuales que ayudarán a la lectura (41); en tercer lugar, hacer hipótesis y/o predicciones sobre el contenido y activar conocimientos previos (37) y, por último, determinar y reconocer las estructuras textuales (14). En general, sobre las estrategias de lectura los estudiantes indicaron que les fueron útiles para contextualizar (52) y para despertar el interés sobre la obra (20). 
Por lo que se refiere a la fase de control, las tareas más lucrativas, por orden de importancia, fueron las siguientes: detectar información relevante y secundaria (68); identificar palabras o estructuras que necesitan ser aclaradas, tomando notas o subrayando (56); contestar a preguntas sobre el texto (53); parafrasear, resumir y sintetizar (44); anticipar información (17); comprobar hipótesis y realizar nuevas inferencias deductivas (9); y generar nuevas preguntas (6).

A la cuestión sobre si las actividades de lectura realizadas les resultaron ventajosas para tomar consciencia sobre el proceso lector, 30 discentes estaban parcialmente de acuerdo y 42 totalmente de acuerdo.

Sobre la utilidad del momento de evaluación de la CL tras la lectura, 69 respuestas manifestaron estar totalmente de acuerdo en que se trata de una fase imprescindible. Las actividades más eficaces durante esta fase fueron: primero, responder a preguntas sobre el texto leído, para evaluar lo aprendido y las estrategias utilizadas (59) y, después, pensar sobre las estrategias que mejor ayudaron a comprender el texto (13).

En general, la mayoría de los informantes señalaron que antes de la puesta en práctica de los ejercicios de CL no conocían las estrategias de pre y poslectura (69). No obstante, ya habían utilizado algunas estrategias desarrolladas durante la lectura (53).

Por último, 62 estudiantes indicaron que el recurso a las estrategias de lectura les sirvió para reflexionar y evaluar conocimientos (62); aprender cultura (55) y aprender léxico (52).

En suma, de la observación de los datos expuestos, concluimos que la percepción sobre el proceso de CL sufre un cambio positivo antes y después de la instrucción docente explícita; la lectura todavía sigue siendo considerada una destreza fácil de desarrollar y, por eso, poco trabajada en el aula, situándose por detrás de la expresión oral y escrita; los textos más trabajados en clase son los divulgativos y, por último, los publicitarios y literarios. La mayor parte de los encuestados afirma no haber trabajado las estrategias metacognitivas durante la preparación y evaluación de la compresión. No obstante, dicen conocer alǵunas estrategias realizadas durante la lectura, como identificar palabras o estructuras y responder a preguntas sobre el texto.

Tras la práctica de la lectura, los discentes reconocen que es necesario el uso y el desarrollo de estrategias para la CL (Brown, Palinscar \& Ambruser, 2006). Al mismo tiempo, el proceso lector adquiere un carácter dinámico, activo y reflexivo (Calero, 2012) que permite a los alumnos desarrollar destrezas lingüuísticas y habilidades estratégicas individuales (Puente, 1991).

Por lo expuesto, podemos afirmar que se ha producido una evolución positiva en la manera de entender la compresión lectora y su relación con los procesos y estrategias metacognitivas, lo que confirma que se han cumplido los objetivos planteados en esta investigación. 


\section{Conclusiones}

La discusión de los datos presentados en este trabajo nos permite comparar los resultados obtenidos con la información procedente de una investigación similar, realizada en el nivel intermedio de ELE. Las principales diferencias, analizando las respuestas de los informantes, se prenden, por un lado, con el hecho de que en el nivel B2 las destrezas practicadas con mayor frecuencia son la comprensión y la expresión oral, en cambio, la expresión escrita se trabaja más en el nivel C1 que la comprensión auditiva. Por otro lado, los tipos de texto utilizados en B2 son los divulgativos y los descriptivos no literarios. Sin embargó, en C1 ya encontramos una mayor variedad de géneros como, por ejemplo, discursos científicos, expositivos o publicitarios, aunque los divulgativos y los argumentativos siguen siendo los más recurrentes.

Asimismo, un dato significativo es que, en el nivel B2, la totalidad de los informantes manifiesta que nunca había utilizado estrategias para trabajar la comprensión lectora. Por el contrario, en el nivel C1, seis informantes declaran que habían empleado alguna de las estrategias.

En lo que se refiere al proceso lector, los discentes B2 nunca han hecho actividades de prelectura, mientras que los de C1 afirman haber hecho actividades de planificación (seis alumnos). En ambos niveles son frecuentes las actividades de control, con la diferencia de que en B2 las estrategias se limitan al subrayado de palabras que no se entienden. También, en ambos niveles, se efectúan actividades de poslectura pero, de nuevo, en los cursos C1 la tipología de ejercicios es de mayor riqueza.

A su vez, entre los resultados de aprendizaje derivados de la práctica lectora, llama la atención que los informantes B2 señalen, en primer lugar, la adquisición de conocimientos léxicos y culturales; en segundo lugar, la reflexión sobre los contenidos lingüuísticos y, después, las habilidades estratégicas. No obstante, en el nivel C1 también se alude a la sintaxis, a la gramática y al desenvolvimiento de la creatividad.

Los alumnos de nivel superior recurren a un mayor número de estrategias metacognitivas en las fases de la preparación y evaluación de la lectura. Al mismo tiempo, aunque en ambos niveles se señala la reflexión y evaluación sobre lo aprendido en la CL como la actividad más relevante de la poslectura, los discentes C1 sitúan a continuación el aprendizaje de la cultura y, posteriormente, del léxico, mientras que los alumnos B2 destacan el aprendizaje del léxico seguido de la cultura. Este hecho se debe a las características y el contenido de las obras trabajadas en el aula, puesto que el libro de María Dueñas incide sobre aspectos culturales muy cercanos a la realidad histórica de los estudiantes portugueses, lo que motivó una mayor reflexión sobre los hechos culturales e históricos entre España y Portugal. 
En general, los resultados de la investigación y las actividades efectuadas en el aula muestran diferencias entre los niveles analizados, relacionadas con la madurez lingüústica de los estudiantes y su capacidad para vincular la información con la cultura de la lengua que aprenden. No obstante, el uso de textos literarios sigue siendo precario y superficial; la competencia lectora continúa releǵada a un segundo plano (Llopis, 2008); los alumnos no recurren a estrateǵias metacognitivas de lectura y en el nivel C1 son poco comunes las actividades antes y durante la lectura.

En definitiva, hemos comprobado que, desde el inicio de la investigación hasta el fin, se produjo un cambio positivo en la actitud y el modo de entender y comprender la lectura por parte de los informantes. Este cambio pone de manifiesto la efectividad de la aplicación de las estrategias en la lectura de discursos narrativos y demuestra que el objetivo de nuestro trabajo ha sido cumplido, permitiéndonos hacer un diagnóstico general sobre la utilización de estrategias metacognitivas en la CL de textos literarios narrativos en el aula de ELE en los niveles B2 y C1 de lengua del ǵrado en Lenǵuas Modernas de la Facultad de Letras de la Universidad de Coímbra.

Así pues, concluimos que los conocimientos previos del lector, sus habilidades cognitivas y estratégicas y las características del texto son elementos principales que inciden y condicionan la comprensión lectora (Calero, 2012; Richter \& Rapp, 2014). Por tanto, defendemos la lectura de textos literarios narrativos como una práctica motivadora (Albaladejo García, 2007) y el aprendizaje significativo del alumno. Confirmamos, así, la hipótesis de que la metacognición y el dominio de las estrategias metacognitivas tienen un peso fundamental en la instrucción de la L2 y la LE (Nunan, 1996; Sandoval Zúñiga et al., 2010). Esperamos que nuestro trabajo sirva para crear actividades efectivas de CL y desarrollar futuras investigaciones de mayor extensión.

\section{Referencias}

Albaladejo García, M. D. (2007). Cómo llevar la literatura al aula de ELE: de la teoría a la práctica. MarcoELE, Revista de didáctica ELE, 5, 1-51. https://marcoele.com/ como-llevar-la-literatura-al-aula-de-ele-de-la-teoria-a-la-practica

Aragón de Moreno, A. (2014). La metacognición como herramienta en la lectura de textos universitarios. Acción pedagógica, 23, 38-46.

Aznar Juan, M. L., y Gamazo Carretero, E. (2019). Estrategias metacognitivas de comprensión lectora en textos literarios. En M. López Pérez, y G. Maya Retamar (Eds.). Lectura y educación literaria (1. ${ }^{\mathrm{a}}$ ed., pp. 15-27). Diputación de Badajoz. http://dx.doi.org/10.14195/978-989-26-1231-7 
Baker, 1., y Brown, A.L. (1984). Metacognitive skills and reading. En D.Pearson (Ed.), Handbook of reading research (1. ${ }^{a}$ ed., pp. 357-394). Longman.

Binkley, M., Rust, K., y Williams, T. (Eds.). (1997). Reading literacy in an international perspective. (1. ${ }^{\mathrm{a}}$ ed.). US Department of Education.

Brown, A.L., Palinesar, A.S., y Armbruster, B.B. (2006). Instruction comprehension-fostering activities in interactive learning situation. En R. B. Ruddell y N. J. Unrau (Eds.). Theoretical Models and Pro-cesses of Reading (5. ${ }^{a}$ ed., pp. 780-809). International Reading Association.

Calero, A. (2012). El lector como reparador de significado. Un ejemplo práctico de instrucción directa en estrategias metacognitivas. Didáctica. Lengua y Literatura, 25, 83-115. http://dx.doi.org/10.5209/rev_DIDA.2013.v25.42237

Cerchiaro Ceballos, E., Paba Barbosa, C., y Sánchez Castellón, L. (2011). Metacognición y comprensión lectora: una relación posible e intencional. Duazary, 8(1), 99 111. https://doi.org/10.21676/2389783X.258

Colomer, T., Manresa, M., Ramada Prieto, L., y Reyes López, L. (2018). Narrativas literarias en educación infantil y primaria. (1. ${ }^{\mathrm{a}} \mathrm{ed}$.). Síntesis.

Consejo de Europa (2002). El Marco común europeo de referencia para las lenguas: aprendizaje, enseñanza, evaluación. (1. ${ }^{a}$ ed.). Anaya y CVC.

Delmastro, A. L. (2010). El andamiaje metacognitivo en contextos de aprendizaje de una lengua extranjera. Didáctica. Lengua y Literatura, 22, 93-124.

Flavell, J. H. (1997). El desarrollo cognitivo (2. ${ }^{\text {a }}$ ed.). Prentice Hall.

González Fernández, A. (1992). Estrategias metacognitivas en la lectura (1. ${ }^{a}$ ed.). Editorial de la Universidad Complutense de Madrid.

Gutiérrez-Braojos, C., y Salmerón Pérez, H. (2012). Estrategias de comprensión lectora: enseñanza y evaluación en educación primaria. Profesorado. Revista de currículum y formación del profesorado, 16(1), 183-202. http://www.ugr. es/ recfpro/rev161ART11.pdf

Heit, I. (2012). Estrategias metacognitivas de comprensión lectora y eficacia en la asiǵnatura de Lengua y Literatura. Revista de Psicología, 8(15), 79-96. https://repositorio.uca.edu.ar/handle/123456789/6016

Irrazábal, N., y Sáux, G. (2005). Comprensión de textos expositivos. Memoria y estrategias lectoras. Educación, Lenguaje y Sociedad, I(3), 33-55.

Jiménez Pérez, E. (Eds.). (2015). La comprensión y la competencia lectoras. (1. ${ }^{\mathrm{a}}$ ed.). Síntesis.

Llopis, R. (2008). La comprensión lectora, ¡esa gran incomprendida! Foro de profesores de E/LE, 4, 1-9. https://doi.org/10.7203/foroele.0.6545

López Delgado M. (2005). Estrategias de comprensión (1. a ed.). Ediciones de la UCLM.

López González, A. (2020). Proyectos de lectura de obras literarias para alumnos de español como lengua extranjera. Tonos Digital; 38, 1-24. http://www.tonosdigital. es/ojs/index.php/tonos/article/view/2389/1109

López Jiménez, G., y Arciniegas Lagos, E. (2004). Metacognición, lectura y construcción de conocimiento. El papel de los sujetos en el aprendizaje significativo. (1. ${ }^{a}$ ed.). Cátedra UNESCO para la lectura y la escritura en América Latina.

Moreno Fernández, F. (1990). Metodología sociolingüústica. (1.ª ed.). Gredos. 
Moreno, C. (2011). Materiales, estrategias y recursos para la enseñanza del español como L2. (1.. ed.). Arco/libros.

Nikolajeva, M. (2014). Reading for learning: Cognitive approaches to children's litera-

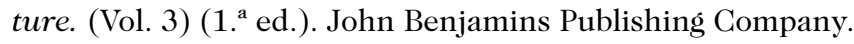

Nunan, D. (1996). Learner strategy training in the classroom: An action research study. TESOL Journal, 6, 35-41.

OCDE (2017). Marco de Evaluación y de Análisis de PISA para el Desarrollo: lectura, matemáticas y ciencias, Versión preliminar. OECD Publishing. https:// www.oecd.org/pisa/aboutpisa/ebook\%20-\%20PISA-D\%20Framework_PRELIMINARY\%20version_SPANISH.pdf

Perfetti, G.A. (2007). Reading ability: Lexical quality to comprehension. Scientific Studies of Reading, 11, 357-383. https://doi. orǵ/10.1080/10888430701530730

Puente, A. (1991). Teoría del esquema y comprensión de lectura. En A. Puente. (Ed.). Comprensión de la lectura y acción docente (1.a ed., pp. 73-109). Pirámide.

Puente, A., Mendoza-Lira, M., Calderón J. F., y Zúñiga, C. (2019). Estrategias metacognitivas lectoras para construir el significado y la representación de los textos escritos. Ocnos, 18(1), 21-30. https://doi.org/10.18239/ocnos_2019.18.1.1781

Ramírez Peña, P., Rossel Ramírez, K., y Nazar Carter, G. (2015). Comprensión lectora y metacognición: Análisis de las actividades de lectura en dos textos de estudio de la asignatura de Lenguaje y Comunicación de séptimo año básico. Estudios Pedagógicos, XLI(2), 213-231. http://dx.doi.org/10.4067/S071807052015000200013

Richards, J. C., y Lockart, Ch. (1998). Estrategias de reflexión sobre la enseñanza de idiomas. (1. ${ }^{\mathrm{a}}$ ed.). Cambridge University Press.

Richards, J., Platt, J., y Platt, H. (1997). Diccionario de lingüústica aplicada y enseñanza de lenguas. (1. ${ }^{\mathrm{a}}$ ed.). Ariel.

Richter, T., y Rapp, D.N. (2014). Comprehension and validation of text information: Introduction to the special issue. Discourse Processes, 51, 1-6. https://doi.org/10. 1080/0163853X.2013.855533

Romero, M.F., Trigo, E., y Moreno, P. (2018). De la comprensión lectora a la competencia literaria a través de la obra de Eliacer Cansino. Ocnos, 17(3), 68-85. https://doi. org/10.18239/ocnos_2018.17.3.1776

Sandoval Zúñiga, M. S., Gómez Álvarez, L., y Sáez Carrillo, K. (2010). Estrateǵias metacognitivas en la comprensión auditiva del inglés como segunda lengua. Lenguas Modernas, 36, 25-44. https://lenguasmodernas.uchile.cl/index.php/LM/article/ view/30679/32435

Solé, I. (1998). Estrategias de lectura. La enseñanza de estrategias de comprensión lectora. (8. ${ }^{a}$ ed.). Graó.

Vidal-Abarca, E., Maña, A., y Gil, L. (2010). Individual differences for self-regulating task-oriented reading activities. Journal of Educational Psychology, 102(4), 817-826. https://doi.org/10.1037/a0020062

Winograd, P. N., y Hare, V. C. (1988). Direct instruction of reading Comprehension strategies: The role of teacher explanation. En E. Goetz, P. Alexander y C. Weinstein. (Eds.). Learning and study strategies: Issues in assessment, instruction 
and evaluation (1. ${ }^{\mathrm{a}}$ ed., pp. 129-139). Academic Press. https://doi.org/10.1016/ B978-0-12-742460-6.50014-1

Zunshine, L. (Ed.). (2015). The Oxford handbook of cognitive literary studies. (1. ${ }^{a}$ ed.). Oxford University Press. https://doi.org/10.1093/oxfordhb/9780199978069.001.0001

Zwaan, R. A., y Singer, M. (2003). Text Comprehension. En A. Graesser, M. A. Gernsbacher y S. R. Goldman (Eds.), Handbook of Discourse Processes (1. ${ }^{\text {a }}$ ed., pp. 83-121). Lawrence Erlbaum. https://www.routledgehandbooks.com/ doi/10.4324/9781410607348.ch3 
